\title{
Numerical study of the phase slips in ultrathin doubly connected superconducting cylinders
}

\author{
Chunyin Qiu ${ }^{1}$ and Tiezheng Qian ${ }^{2, *}$ \\ ${ }^{1}$ Program in Nano Science and Technology, Hong Kong University of Science and Technology, Clear Water Bay, \\ Kowloon, Hong Kong, China \\ ${ }^{2}$ Department of Mathematics, Hong Kong University of Science and Technology, Clear Water Bay, Kowloon, Hong Kong, China
}

(Received 22 October 2008; published 11 February 2009)

\begin{abstract}
In the framework of Ginzburg-Landau theory, we numerically investigate the thermally activated phase slips which are responsible for the current dissipation in ultrathin doubly connected superconducting cylinders in the presence of transport current and external magnetic field along the cylinder axis. A hollow cylinder of radius $R$ is mathematically transformed into a two-dimensional (2D) superconducting strip of width $w=2 \pi R$ with periodic boundary condition. The phase slips may occur via free-energy saddle points of two distinct kinds. The saddle points of the first kind exhibit a one-dimensional (1D) variation of order parameter described by the (extended) Langer-Ambegaokar-McCumber-Halperin (LAMH) theory [Phys. Rev. 164, 498 (1967); Phys. Rev. B 1, 1054 (1970)]. The saddle points of the second kind exhibit a 2D variation of order parameter, showing that each phase slip is realized through a thermally activated process of vortex-antivortex pair creation and annihilation. In particular, there exists a critical radius $R_{c}$ separating the 1D LAMH behavior (below $R_{c}$ ) and the $2 \mathrm{D}$ vortex-antivortex behavior (above $R_{c}$ ). The effects of external magnetic field on these saddle points are presented. The critical radius $R_{c}$ is found to decrease with increasing field strength, and hence applying a magnetic field may induce a transition in the phase-slip characteristics.
\end{abstract}

DOI: 10.1103/PhysRevB.79.054513

PACS number(s): 74.40.+k, 74.20.De, 82.20.Wt, 05.10.-a

\section{INTRODUCTION}

Suppression of superconductivity by fluctuations in lowdimensional systems has been of theoretical and experimental interest for decades. In one-dimensional (1D) systems, i.e., wires, thermally activated phase slips are responsible for the decay of supercurrent at temperatures below the critical temperature $T_{c}$, according to a series of theoretical works by Little, ${ }^{1}$ Langer and Ambegaokar (LA), ${ }^{2}$ and McCumber and Halperin (MH). ${ }^{3}$ The LAMH theory ${ }^{2,3}$ provides a framework for quantitative studies of the current dissipation in superconducting wires below $T_{c}$. In two-dimensional (2D) superconducting thin films that are wide enough, the current dissipation is due to the flow of free vortices induced by the thermally activated (and/or current assisted) unbinding of vortex-antivortex pairs (VAPs), quantitatively described by the resistance theory based on the Kosterlitz-Thouless transition. ${ }^{4-10}$ The above 1D (Refs. 1-3 and 11-13) and 2D (Refs. 4-10) problems have been extensively investigated both theoretically and experimentally. However, the case of intermediate dimension has received relatively less attention. Recently, the mechanism of current dissipation in quasi-2D narrow superconducting strips has been investigated. ${ }^{14-16} \mathrm{We}$ have numerically shown that the current dissipation in quasi-2D strips may arise from phase slips that involve two distinct kinds of saddle points of the free-energy functional. ${ }^{16}$ A critical value of strip width $w_{c}$ is numerically determined. Below $w_{c}$, the strip behaves as a 1D superconducting wire in which the phase slips are described by the LAMH theory. Above $w_{c}$, however, the $2 \mathrm{D}$ character of the strip is recovered: each phase slip is realized through a single vortex crossing the strip.

Similar to a quasi-2D superconducting strip, an ultrathin doubly connected superconducting cylinder (DCSC) constitutes another link between 1D and 2D superconductors. One of the most important properties of the cylindrical geometry is the Little-Parks oscillation of the critical temperature with the magnetic flux piercing the cylinder. ${ }^{17-19}$ In particular, the novel destructive regime near half-integer flux quanta (in which there is no superconducting condensation at zero temperature) has been investigated in ultrathin DCSCs. ${ }^{20-22} \mathrm{~A}$ phase-separation mechanism ${ }^{21,22}$ has been proposed for the observed temperature dependence of resistance, but the current dissipation in ultrathin DCSCs remains an unresolved issue. Conceptually, a hollow superconducting cylinder can be viewed as a rolled-up 2D strip. Compared to a usual "flat" superconducting strip, the cylindrical geometry means that transverse (circumferential) persistent current can be induced by a magnetic field piercing the cylinder. For temperatures below $T_{c}$ and transport currents below the critical current, thermally activated phase slips may still be responsible for the current dissipation in ultrathin DCSCs, but under the influence of field-induced persistent current. In this paper, we present a numerical study of these phase slips, with a focus on the quantitative effects of applied magnetic field.

The paper is organized as follows. The theoretical model is presented in Sec. II. The ultrathin DCSC is described by a Ginzburg-Landau (GL) free-energy functional and the corresponding time-dependent GL equation with a Langevin noise. For the convenience of presentation, the DCSC is transformed into a mathematically equivalent geometry, i.e., a 2D strip with appropriate boundary conditions. The metastable current-carrying states, free-energy saddle points, and current-reducing phase-slip fluctuations are then described. The numerical results are presented in Sec. III. There exist two distinct kinds of phase slips corresponding to two distinct kinds of free-energy saddle points. The phase slips of the first kind are of $1 \mathrm{D}$ character numerically observed for cylinder radius below a critical value. Those of the second kind are of $2 \mathrm{D}$ character observed for cylinder radius above the critical value. The effects of the external magnetic field 
(a)

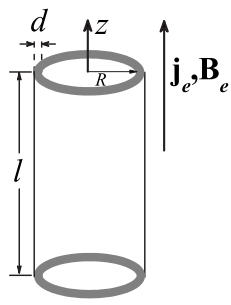

(b)

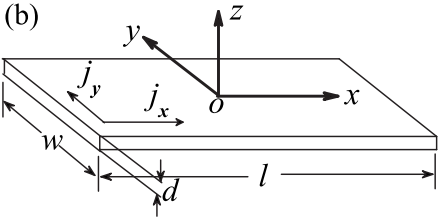

FIG. 1. (a) Schematic illustration of an ultrathin DCSC of radius $R$, length $l$, and thickness $d$, with the external magnetic field $\mathbf{B}_{e}$ and transport current of density $\mathbf{j}_{e}$ applied along the cylinder axis, i.e., the $z$ direction. (b) Schematic illustration of the corresponding superconducting strip of width $w=2 \pi R$, with $x$ and $y$ coordinates corresponding to $z$ and $R \theta$ in (a), respectively.

piercing the cylinder are investigated, with the critical radius found to decrease with increasing field strength. The paper is concluded in Sec. IV.

\section{PHASE-SLIP FLUCTUATIONS IN ULTRATHIN DOUBLY CONNECTED SUPERCONDUCTING CYLINDERS}

\section{A. Ultrathin DCSC}

Figure 1(a) is a schematic illustration of an ultrathin DCSC. The radius, length, and thickness of the hollow cylinder are denoted by $R, l$, and $d$, respectively. The external magnetic field $\mathbf{B}_{e}$ and transport current of density $\mathbf{j}_{e}$ are both applied along the axis of cylinder, i.e., the $z$ direction. For the ultrathin DCSC considered here, the thickness $d$ is assumed to be much smaller than the temperature-dependent correlation length and penetration length. The variations of the order parameter $\psi$ along the radial direction are energetically prohibited and the self-induced magnetic field is negligible. (Typically, we consider $R$ of several temperature-dependent correlation lengths and assume $R \gg d$, so that there is no need to distinguish the outer and inner radii.) The GL free-energy functional is of the form

$$
F[\psi]=d \int d S\left[\frac{K}{2}\left|\left(\nabla-\frac{i e^{*} \mathbf{A}}{\hbar c}\right) \psi\right|^{2}-\frac{\alpha}{2}|\psi|^{2}+\frac{\beta}{4}|\psi|^{4}\right],
$$

where $K=\hbar^{2} / m$, $\hbar$ is the Planck's constant, and $m$ is the effective mass of a Cooper pair, $e^{*}=2 e, \mathbf{A}$ is the vector potential, $\alpha=\alpha_{0}\left(T_{c}-T\right)$, and $\alpha_{0}$ and $\beta$ are both phenomenological material parameters. The integration over the cylinder surface is represented by $\int d S=R \iint d z d \theta$, with $\theta$ being the azimuthal angle in cylindrical coordinate system. It is convenient to choose the symmetric gauge $\mathbf{A}=\mathbf{B}_{e} \times \mathbf{r} / 2$ and hence $\mathbf{A}=B_{e} R \hat{\boldsymbol{\theta}} / 2$, with $\hat{\boldsymbol{\theta}}$ being the unit vector in the azimuthal direction. Mathematically, a hollow cylinder of radius $R$ is equivalent to a $2 \mathrm{D}$ strip of width $w=2 \pi R$ supplemented with the periodic boundary condition (PBC) along the $\hat{\boldsymbol{\theta}}$ direction. For the convenience of presentation, we make the coordinate transformation $z \rightarrow x$ and $R \theta \rightarrow y$ to construct a new $x y$ coordinate system, in which the equivalent $2 \mathrm{D}$ strip has its center located at the origin, as shown in Fig. 1(b). In the $x y$ system, the current density $\mathbf{j}_{e}$ becomes $j_{x} \hat{\mathbf{x}}$ and the vector potential A becomes $A \hat{\mathbf{y}}$ which induces a persistent current of density $j_{y} \hat{\mathbf{y}}$, to be detailed below, with $\hat{\mathbf{x}}$ and $\hat{\mathbf{y}}$ being the unit vectors in the $x$ and $y$ directions.

The time evolution of $\psi$ is governed by the timedependent GL equation with a white noise,

$$
\begin{aligned}
\gamma \frac{\partial \psi}{\partial t}= & -\frac{1}{d} \frac{\delta F[\psi]}{\delta \psi^{*}}+\zeta \\
= & K\left[\nabla^{2} \psi-\left(2 i e^{*} / \hbar c\right) \mathbf{A} \cdot \nabla \psi-\left(e^{*} A / \hbar c\right)^{2} \psi\right] \\
& +\alpha \psi-\beta|\psi|^{2} \psi+\zeta,
\end{aligned}
$$

where $\gamma$ is the damping coefficient, the asterisk denotes the complex conjugate, and $\zeta(x, y, t)$ is a white noise satisfying the autocorrelation functions

$$
\begin{gathered}
\left\langle\zeta(x, y, t) \zeta\left(x^{\prime}, y^{\prime}, t^{\prime}\right)\right\rangle=0, \\
\left\langle\zeta(x, y, t) \zeta^{*}\left(x^{\prime}, y^{\prime}, t^{\prime}\right)\right\rangle=4 d^{-1} \gamma k_{B} T \delta\left(x-x^{\prime}\right) \delta\left(y-y^{\prime}\right) \delta\left(t-t^{\prime}\right) .
\end{gathered}
$$

This noise generates a random motion of $\psi(x, y)$ in the configuration space and stabilizes an equilibrium distribution with the probability density proportional to $e^{-F[\psi] / k_{B} T}$.

For computational purpose, we use the dimensionless form

$$
\bar{F}[\bar{\psi}]=\int d \bar{x} d \bar{y}\left[\frac{1}{2}|(\bar{\nabla}-i \overline{\mathbf{A}}) \bar{\psi}|^{2}-\frac{1}{2}|\bar{\psi}|^{2}+\frac{1}{4}|\bar{\psi}|^{4}\right]
$$

for the free-energy functional. Here the overbar denotes the dimensionless quantities obtained with $F$ scaled by $G(T)$ $=d \xi^{2} \alpha^{2} / \beta, \quad \psi$ by $\sqrt{\alpha / \beta}, x$ and $y$ by the temperaturedependent correlation length $\xi=\sqrt{K / \alpha}$, and the vector potential A by $\boldsymbol{\Phi}_{o} / 2 \pi \xi$ with $\boldsymbol{\Phi}_{o}=2 \pi \hbar c / e^{*}$ being the flux quantum. The magnetic flux piercing the cylinder $\boldsymbol{\Phi}=\pi R^{2} B_{e}$ is a physical quantity measuring the strength of applied field. Scaling the applied flux $\boldsymbol{\Phi}=\pi R^{2} B_{e}$ by $\boldsymbol{\Phi}_{o}$ yields the dimensionless flux $\overline{\boldsymbol{\Phi}}=\boldsymbol{\Phi} / \boldsymbol{\Phi}_{o}$ with the relation $\overline{\boldsymbol{\Phi}}=\bar{A} \bar{R}$. The dimensionless equation corresponding to Eq. (2) is of the form

$$
\frac{\partial \bar{\psi}}{\partial \bar{t}}=-\frac{\delta \bar{F}[\bar{\psi}]}{\delta \bar{\psi}^{*}}+\bar{\zeta}=\bar{\nabla}^{2} \bar{\psi}-2 i \overline{\mathbf{A}} \cdot \bar{\nabla} \bar{\psi}+\left(1-\bar{A}^{2}-|\bar{\psi}|^{2}\right) \bar{\psi}+\bar{\zeta},
$$

in which the time is scaled by $\tau(T)=\gamma / \alpha$, i.e., $\bar{t}=t / \tau(T)$, and the dimensionless noise $\bar{\zeta}$ satisfies the autocorrelation functions

$$
\begin{gathered}
\left\langle\bar{\zeta}(\bar{x}, \bar{y}, \bar{t}) \bar{\zeta}\left(\bar{x}^{\prime}, \bar{y}^{\prime}, \bar{t}^{\prime}\right)\right\rangle=0, \\
\left\langle\bar{\zeta}(\bar{x}, \bar{y}, \bar{t}) \bar{\zeta}^{*}\left(\bar{x}^{\prime}, \bar{y}^{\prime}, \bar{t}^{\prime}\right)\right\rangle \\
=4\left[k_{B} T / G(T)\right] \delta\left(\bar{x}-\bar{x}^{\prime}\right) \delta\left(\bar{y}-\bar{y}^{\prime}\right) \delta\left(\bar{t}-\bar{t}^{\prime}\right) .
\end{gathered}
$$

Throughout the remainder of this paper, we mostly use the dimensionless quantities with the overbar dropped. We want to point out that all the temperature effects have been absorbed into the units [e.g., $G(T), \sqrt{\alpha / \beta}$, and $\xi]$ in defining the dimensionless quantities. 


\section{B. Metastable states, saddle points, and phase slips}

The metastable current-carrying states are the local minima of the GL free-energy functional, which can be obtained from the stationary GL equation

$$
\nabla^{2} \psi-2 i \mathbf{A} \cdot \nabla \psi+\left(1-A^{2}-|\psi|^{2}\right) \psi=0 .
$$

It is convenient to take $\psi(x, y)=f(x, y) e^{i \phi(x, y)}$, in which $f$ and $\phi$ represent the magnitude and phase of the complex order parameter. Then, Eq. (5) becomes

$$
\nabla^{2} f-f \nabla \phi \cdot \nabla \phi+2 f \mathbf{A} \cdot \nabla \phi+\left(1-A^{2}-f^{2}\right) f=0
$$

and

$$
\nabla \cdot\left[f^{2}(\nabla \phi-\mathbf{A})\right]=\nabla \cdot \mathbf{j}=0,
$$

in which $\mathbf{j}=f^{2}(\nabla \phi-\mathbf{A})$ is the (dimensionless) superconducting current density. The GL equations (6a) and (6b) can be solved under appropriate boundary conditions. Besides the PBC along the $y$ direction $\psi(x,-w / 2)=\psi(x, w / 2)$, which is required to transform the hollow cylinder into the strip, the PBC is also applied in the $x$ direction, with $\psi(-l / 2, y)$ $=\psi(l / 2, y)$. Under these boundary conditions, the metastable states are of uniform magnitude, i.e., $\nabla f=0$, and the solutions of Eqs. (6a) and (6b) are of the form

$$
\psi_{n}(x, y)=f_{n} e^{i k_{n} x},
$$

where $k_{n}=2 n \pi / l$ is the wave vector along the $x$ direction, $f_{n}=\sqrt{1-A^{2}-k_{n}^{2}}$ is the constant magnitude, and $n$ is an integer usually called the winding number. Physical observables are periodic functions of the applied magnetic flux $\boldsymbol{\Phi}$. Here we only consider $0 \leq \boldsymbol{\Phi} \leq 1 / 2$ and assume a zero winding number in the $y$ direction (to minimize the free-energy contribution from order-parameter variation in that direction). The current density now has two components: the longitudinal $x$ component $j_{x}=f_{n}^{2} k_{n}$ from the transport current and the transverse $y$ component $j_{y}=-f_{n}^{2} A$ from the persistent current induced by the external magnetic field. In the presence of an external field, $\psi_{n}$ remains metastable if the magnitude of wave vector $\left|k_{n}\right|$ does not exceed the critical value $k_{c}$ $=\sqrt{\left(1-A^{2}\right) / 3}$, which corresponds to the critical current density $j_{x c}=2\left[\left(1-A^{2}\right) / 3\right]^{3 / 2}$. As expected, $j_{x c}$ decreases with increasing external field.

Between two neighboring metastable states $\psi_{n}$ and $\psi_{n-1}$, there is a saddle point of the GL free-energy functional, $\psi_{s}(x, y)$, which is also a solution of the stationary GL equation. Physically, this saddle point corresponds to the most probable thermally activated fluctuation which can carry the system from $\psi_{n}$ to $\psi_{n-1}$ and vice versa. The free-energy barrier $\Delta F$ is given by $\Delta F=F_{s}-F_{n}\left(\Delta F=F_{s}-F_{n-1}\right)$ for the transition $\psi_{n} \rightarrow \psi_{n-1}\left(\psi_{n-1} \rightarrow \psi_{n}\right)$, with $F_{n}\left(F_{n-1}\right)$ and $F_{s}$ denoting the free energies of $\psi_{n}\left(\psi_{n-1}\right)$ and $\psi_{s}$, respectively. According to the free-energy expression $F_{n}=-\left(1-A^{2}-k_{n}^{2}\right)^{2} w l / 4$, a smaller winding number (corresponding to a weaker transport current) yields a lower free energy. Therefore, the transition $\psi_{n} \rightarrow \psi_{n-1}$ (for positive $n$ ) with a phase change of $-2 \pi$ (over the length $l$ ) is much more probable than the transition $\psi_{n-1} \rightarrow \psi_{n}$ with a phase change of $2 \pi$ since the free-energy barrier $\Delta F=F_{s}-F_{n}$ is smaller. Because of this current-biased barrier crossing, the thermally activated phase slips result in a spontaneous (irreversible) process in which the free energy is lowered and the current is reduced. Based on this observation and also for the convenience of presentation, our discussions will be focusing on the current-reducing transition $\psi_{n} \rightarrow \psi_{n-1}$; the extension to the transition $\psi_{n-1} \rightarrow \psi_{n}$ is straightforward.

For 2D superconducting strips with superconductorinsulator boundary condition $\partial_{y} \psi(x, \pm w / 2)=0$ applied at the two edges $y= \pm w / 2$, we have verified that there exist two distinct kinds of saddle points corresponding to two distinct kinds of phase slips. ${ }^{16}$ Saddle points of the first kind have been called the phase-slip-strip (PSS) solution, ${ }^{15,16}$ in which the superconducting strip acts as a $1 \mathrm{D}$ wire with the order parameter uniformly distributed along the $y$ direction across the strip. The PSS solution $\psi_{s}^{\mathrm{PSS}}$ and the corresponding freeenergy barrier $\Delta F^{\mathrm{PSS}}$ can be quantitatively described by the LAMH theory. Saddle points of the second kind have been called the phase-slip-vortex (PSV) solution, in which vortices are involved and thus the order parameter varies in the two directions. The phase slip occurs through a single vortex (generated at boundary) crossing the strip. The PSV solution $\psi_{s}^{\mathrm{PSV}}$ disappears as the strip width is reduced to a critical value $\cong 4.4,{ }^{16}$ below which the PSS solution is the only channel for phase slips to occur. Above this critical value, however, saddle points of the two kinds coexist with the PSV solution dominating the phase slips because of $\Delta F_{s}^{\mathrm{PSV}}$ $<\Delta F_{s}^{\mathrm{PSS}}$, where $\Delta F_{s}^{\mathrm{PSV}}$ is the free-energy barrier for the PSV solution.

With the PBCs applied in both the $x$ and $y$ directions, the PSS solution remains unchanged since the 1D orderparameter variation satisfies the PBC in the $y$ direction automatically. However, the PSV solution is now prohibited as a single vortex is not allowed to exist alone under the PBCs in both the $x$ and $y$ directions. (The 2D system is mathematically equivalent to a torus, which does not permit the existence of a single vortex.) The vortices may however exist in the form of VAP, which is permitted by the PBCs in 2D space. To be shown in Sec. III is a kind of saddle points that involve the creation and annihilation of a vortex-antivortex pair through which a phase slip may occur. We call this kind of saddle points the phase-slip-VAP (PSVAP) solution, a generalization of PSV solution to cylinders. In the absence of external magnetic field, the PSVAP solution can be obtained simply through a mapping for a given uniform transport current. That is, a strip of width $w$ that contains a VAP and satisfies the PBC in the transverse $(y)$ direction can be divided into two strips of width $w / 2$. Of these two strips, one holds a vortex and the other holds its image, i.e., an antivortex. The superconductor-insulator boundary condition is satisfied at the edges in the transverse direction of each strip. Based on this mapping, the free-energy barrier $\Delta F^{\mathrm{PSVAP}}$ associated with the PSVAP solution for a cylinder of width $w$ is given by $\Delta F^{\mathrm{PSVAP}}(w)=2 \Delta F^{\mathrm{PSV}}(w / 2)$, where $\Delta F^{\mathrm{PSV}}(w / 2)$ is the free-energy barrier associated with the PSV solution for a strip of width $w / 2$. This relation can also be established from the analytical expressions derived in the framework of London theory (see Appendix, Sec. 2 for details). The above mapping suggests that, in the absence of external field, the behavior of phase slips in the DCSC system (with the PBC applied in the $y$ direction) is determined by a critical width 
$w_{c 0} \cong 8.8$, which is twice the critical width $(\cong 4.4)$ found for strips. ${ }^{16}$ [Here the subscript " 0 " indicates zero external field.]

Naturally, it is expected that the saddle points described above, i.e., the PSS solution and the PSVAP solution, would be affected as an external magnetic field is applied, by which a persistent current along the transverse $y$ direction is induced. In Sec. III, we present the numerical results showing the quantitative effects of the applied field on phase slips. As expected, the magnetic field always suppresses the superconductivity: it enhances the current-reducing fluctuations by reducing the free-energy barrier. Because of the $1 \mathrm{D}$ character of the PSS solution, the LAMH theory can be readily extended to obtain $\psi_{s}^{\mathrm{PSS}}$ and the corresponding free-energy barrier $\Delta F^{\mathrm{PSS}}$ analytically (see Appendix, Sec. 1). For the PSVAP solution, however, it would be very difficult to obtain any analytical results in the GL description.

Recently, the string method ${ }^{23-25}$ has been presented for the numerical evaluation of thermally activated rare events. This method first locates the most probable transition pathway connecting two stable/metastable states in configuration space. This is done by evolving strings, which are smooth curves with intrinsic parametrization (such as arc length) in configuration space, toward the minimal-energy path (MEP). Once the MEP is obtained, the saddle point is determined by locating the maximum of energy potential along the MEP. We have demonstrated the string method to be an efficient numerical tool for evaluating the thermally activated phase slips in 1D superconducting wires ${ }^{26,27}$ and quasi-2D narrow superconducting strips. ${ }^{16}$ The extension of this method to the ultrathin DCSC system is straightforward. In particular, by using appropriately prepared initial strings, we can obtain both the PSS solution and the PSVAP solution. The simplest initial string is constructed as a linear interpolation between the metastable states $\psi_{n}$ and $\psi_{n-1}$ [expressed in Eq. (7)] and leads to the PSS MEP (i.e., the MEP along which the saddle point is found to be the PSS solution). Mathematically, a linear interpolation between $\psi_{n}$ and $\psi_{n-1}$ involves only 1D variation of $\psi$ along the $x$ direction, and so does the saddle point $\psi_{s}^{\text {PSS }}$ obtained from such an initial string. In order to obtain the PSVAP MEP (i.e., the MEP along which the PSVAP solution occurs at the saddle point), a small perturbation to the above initial string has to be used to introduce some variation along the $y$ direction as well.

\section{NUMERICAL RESULTS}

Below we present the numerical details for the evaluation of saddle points intervening neighboring metastable states. Without losing generality, we start from the transition $\psi_{3}$ $\rightarrow \psi_{2}$ for a system of length $l=200$ and width $w=16$ (corresponding to a DCSC of radius $R \cong 2.55$ ). As $w>w_{c 0} \cong 8.8$, phase slips may be thermally activated through either the PSS solution or the PSVAP solution. In the absence of external field, the transport current density is found to be $j_{x}$ $\cong 0.0934 \cong 0.24 j_{x c}$, which is moderately strong. When the external field of flux $\boldsymbol{\Phi}=0.2$ is applied, a transverse persistent current of density $j_{y} \cong-0.0774$ is induced and the transport current density is slightly reduced to a value $\cong 0.0928$.
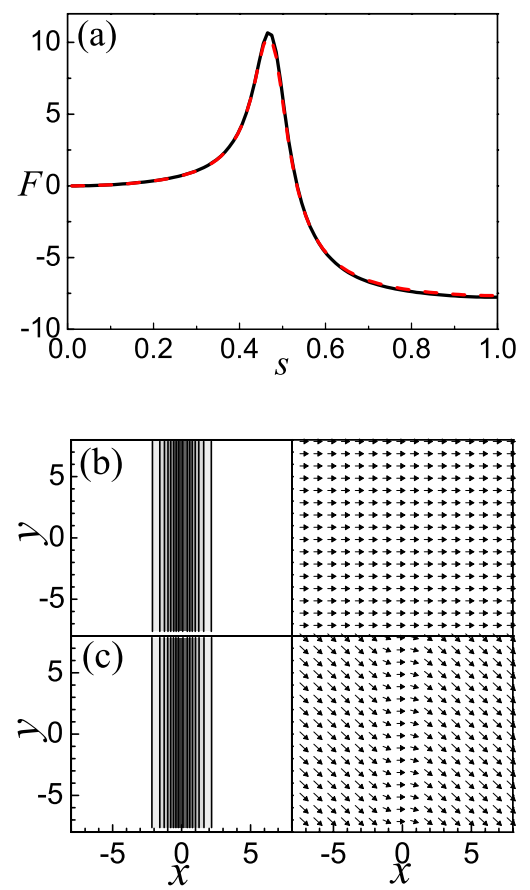

FIG. 2. (Color online) (a) Dimensionless free energy $F$ evaluated along the PSS MEP from $\psi_{3}$ to $\psi_{2}$ plotted as a function of the arc length $s$ in the $\psi(x, y)$-function space, for $l=200$ and $w=16$, with the black solid and red dashed lines representing the $\boldsymbol{\Phi}=0$ and $\boldsymbol{\Phi}=0.2$ cases, respectively. The $\psi_{3}$ state is taken as the reference point at which $s=0$. The arc length measured along the MEP is normalized by that from $\psi_{3}$ to $\psi_{2}$; hence, $s$ runs from 0 to 1 . In each set of the data, the corresponding value at $\psi_{3}$ has been subtracted to let the curve start from zero. (b) Distributions of order-parameter magnitude (left) and current density (right) for the PSS solution at $\boldsymbol{\Phi}=0$. The grayscale varies from black for $|\psi|=0$ to white for $|\psi|$ $=1$. Here only the segments of noticeable spatial variations are shown for clear illustration. (c) The same as (b) obtained for $\boldsymbol{\Phi}$ $=0.2$.

\section{A. Phase slips via PSS solution}

Figure 2(a) shows the free-energy variation along the PSS MEP from $\psi_{3}$ to $\psi_{2}$, evaluated for $\boldsymbol{\Phi}=0$ (solid line) and $\boldsymbol{\Phi}$ $=0.2$ (dashed line), respectively. By locating the free-energy maximum at each curve, we find the free-energy barrier to be $\Delta F^{\mathrm{PSS}}=F\left[\psi_{s}^{\mathrm{PSS}}\right]-F_{3}=10.90$ for $\boldsymbol{\Phi}=0$ and $\Delta F^{\mathrm{PSS}}=10.79$ for $\boldsymbol{\Phi}=0.2$. The latter is slightly suppressed compared to the former. These values are very close to the theoretical predictions of the (extended) LAMH theory (see Appendix, Sec. 1): $\Delta F^{\mathrm{PSS}}=10.92$ for $\boldsymbol{\Phi}=0$ and $\Delta F^{\mathrm{PSS}}=10.80$ for $\boldsymbol{\Phi}=0.2$. Displayed in Figs. 2(b) and 2(c) are the spatial distributions of order-parameter magnitude (left) and current density (right) evaluated at the PSS solution (i.e., the saddle point located at the PSS MEP), both showing clearly the 1D character (with no variation in the $y$ direction). Figures 2(b) and 2(c) also show that the $x$ component of current density $j_{x}$ is a constant in the $x$ direction, as required by Eq. (6b), which becomes $\nabla \cdot \mathbf{j}=\partial_{x} j_{x}=0$ for the present $1 \mathrm{D}$ variation. It is interesting to note that for $\boldsymbol{\Phi}=0.2$ [shown in Fig. 2(c)], the $y$ component of current density $j_{y}$ is not a constant in the $x$ direction (with $j_{y}$ vanishing at the phase-slip center) due to the order-parameter 


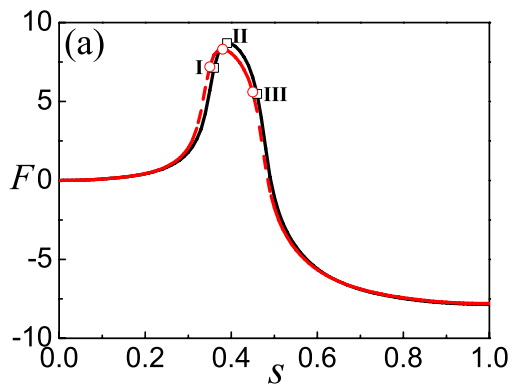

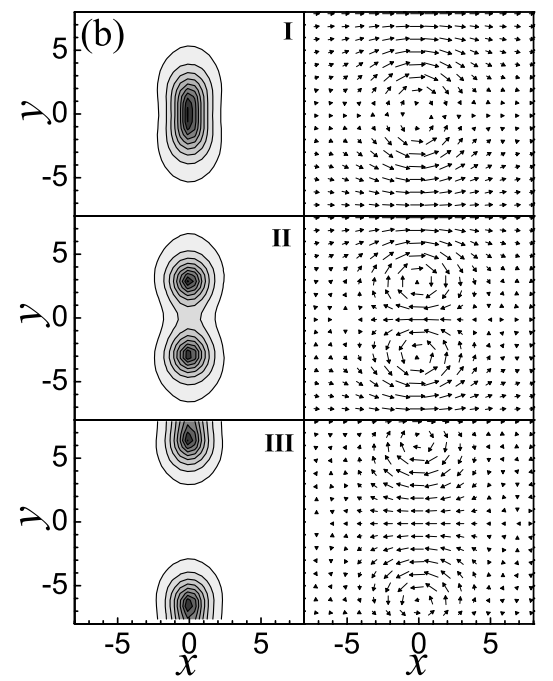

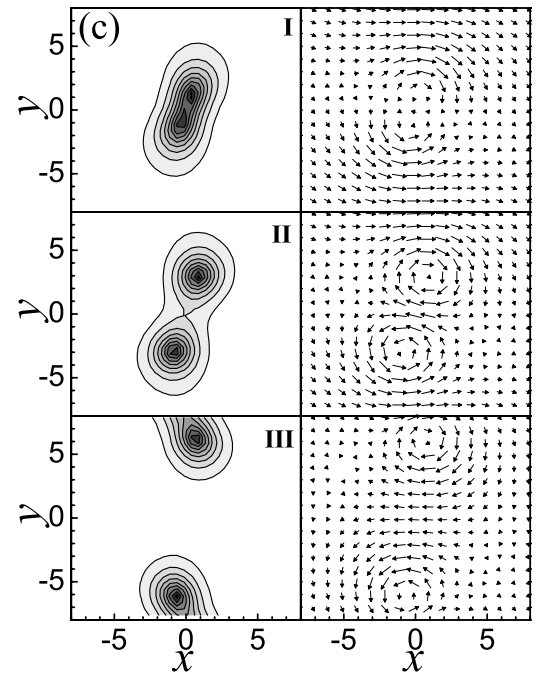

FIG. 3. (Color online) (a) Dimensionless free energy $F$ evaluated along the PSVAP MEP from $\psi_{3}$ to $\psi_{2}$ plotted as a function of the arc length $s$ in the $\psi(x, y)$-function space, with the black solid and red dashed lines representing the $\boldsymbol{\Phi}=0$ and $\boldsymbol{\Phi}$ $=0.2$ cases, respectively. Here $F$ and $s$ are defined in the same way as for Fig. 2(a). (b) Distributions of order-parameter magnitude (left) and current density (right) for a sequence of states labeled along the curve for $\boldsymbol{\Phi}=0$ in (a). The grayscale varies from black for $|\psi|=0$ to white for $|\psi|=1$. Only the segments of noticeable spatial variations are shown for clear illustration. (c) The same as (b) obtained for $\boldsymbol{\Phi}=0.2$. variation in this direction. A phase slip occurs once the saddle point is crossed in a large thermal fluctuation and a segment of superconductor turns normal momentarily, thus allowing the winding number to change by 1 and the phase to slip by $2 \pi$.

\section{B. Phase slips via PSVAP solution}

Now we turn to the saddle points of the second kind. Figure 3(a) shows the free-energy variation along the PSVAP MEP connecting the current-carrying states $\psi_{3}$ and $\psi_{2}$ for the cases of $\boldsymbol{\Phi}=0$ (solid line) and 0.2 (dashed line). The freeenergy barrier associated with the PSVAP solution is evaluated to be $\Delta F^{\mathrm{PSVAP}}=F\left[\psi_{s}^{\mathrm{PSVAP}}\right]-F_{3}=8.70$ for $\boldsymbol{\Phi}=0$ and $\Delta F^{\mathrm{PSVAP}}=8.32$ for $\boldsymbol{\Phi}=0.2$. These values are smaller than those obtained for the PSS solution in Sec. III A, i.e., $\Delta F^{\mathrm{PSVAP}}<\Delta F^{\mathrm{PSS}}$ for both $\boldsymbol{\Phi}=0$ and 0.2 . Therefore, in the strip of width $w=16$, phase slips are dominated by thermal fluctuations through the PSVAP solution, i.e., VAP creation and annihilation. In addition, the free-energy barrier associated with the PSVAP solution is much more susceptible to the applied magnetic field. As $\boldsymbol{\Phi}$ is increased from 0 to 0.2 , $\Delta F^{\mathrm{PSVAP}}$ is suppressed by 0.38 while $\Delta F^{\mathrm{PSS}}$ is suppressed by 0.11. The sensitivity of the PSVAP free-energy barrier $\Delta F^{\mathrm{PSVAP}}$ to external field will be further discussed in Sec. III C.

In the framework of London theory, we have presented an analytical expression of the PSV free-energy barrier $\Delta F^{\mathrm{PSV}}$ for strips with superconductor-insulator boundary condition. ${ }^{16}$ In this approximate description based on the
London theory, the internal structure of the vortex core is neglected and the PSV solution is simply represented by a $2 \mathrm{D}$ position of the vortex center that yields the maximum free energy. Excellent agreement has been achieved for relatively wide strips $(w \geq 10)$, with a positive fitting parameter used for the core energy. Although the London description is oversimplified and inaccurate for narrow strips, it provides a direct picture of the vortex motion in real space. We have developed a London description for the PSVAP solution which dominates in DCSCs of relatively large radius. We find that explicit expression for the PSVAP solution can only be obtained for a few special cases. The string method is also employed to numerically evaluate the PSVAP solution and the associated free-energy barrier in the London description (see Appendix, Sec. 2 for details). For the system investigated above, the free-energy barrier is evaluated to be $\Delta F^{\mathrm{PSVAP}}=8.69$ for $\boldsymbol{\Phi}=0$ and $\Delta F^{\mathrm{PSVAP}}=8.28$ for $\boldsymbol{\Phi}=0.2$. In quantitatively fitting the corresponding values of 8.70 and 8.32 obtained earlier in the framework of GL theory, we use the core energy $E_{c}=1.25$, which is close to the values $E_{c}$ $\cong 1.27$ and 1.22 used in Ref. 16 .

Displayed in Figs. 3(b) and 3(c) are the distributions of order-parameter magnitude (left) and current density (right) evaluated for a sequence of states along the PSVAP MEP from $\psi_{3}$ to $\psi_{2}$. It is shown that a pair of vortex (with clockwise current circulation) and antivortex (with counterclockwise current circulation) is first nucleated at the center in the transverse direction (state I). They are then separated from each other by thermal activation (state II, which is the stationary PSVAP solution) and finally annihilated at the boundaries (state III) where they are recombined (because of the 


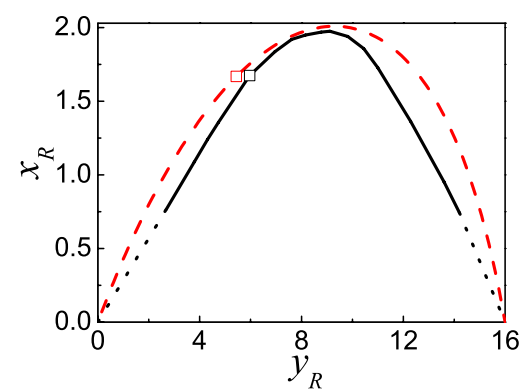

FIG. 4. (Color online) The passage for the motion of the vortex relative to the antivortex at $\boldsymbol{\Phi}=0.2$. The black solid line represents the result obtained in the framework of GL theory and the red dashed line represents the result obtained in the framework of London theory. Here the dotted lines represent an extrapolation of the GL result because the positions of vortex and antivortex become indistinguishable as they are close enough. The squares denote the position of the vortex relative to the antivortex in the PSVAP solution.

PBC in the $y$ direction). Compared to Fig. 3(b) for $\boldsymbol{\Phi}=0$, the orientation of VAP in Fig. 3(c) for $\boldsymbol{\Phi}=0.2$ deviates from the $y$ direction, indicating that the vortex and antivortex are not moving perpendicularly to the strip. To show this fieldinduced feature more clearly, we plot in Fig. 4 the passage $\mathbf{r}_{R}=\left(x_{R}, y_{R}\right)$ for the motion of the vortex relative to the antivortex along the PSVAP MEP for $\boldsymbol{\Phi}=0.2$. The passage obtained in the framework of GL theory is compared with that obtained in the framework of London theory, and a semiquantitative agreement is seen. Physically, the $x$ component of the relative motion arises from the force in the $x$ direction caused by the field-induced $y$ component of current density [see Eq. (A5) in Appendix, Sec. 2].

To see how a phase slip occurs in 2D space, we turn to the phase distribution $\varphi(x, y)$ of the complex order parameter. Without losing generality, we take the PSVAP solution for $\boldsymbol{\Phi}=0.2$ [state II in Fig. 3(c)] as an example. Figure 5(a) shows the contour plot of $\varphi(x, y)$, from which the existence of a vortex and an antivortex is clearly observed. In the space above the vortex and below the antivortex, the phase increases continuously in the $+x$ direction. In the space in between, there is a sudden phase change of $2 \pi$ around $x=0$ (with the color jumped from blue to red). This is shown more

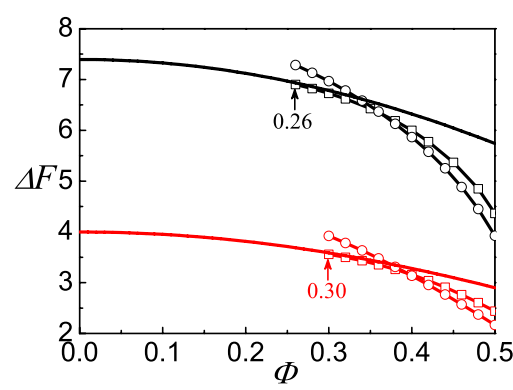

FIG. 6. (Color online) Free-energy barrier plotted as a function of $\boldsymbol{\Phi}$, evaluated for a sample of width $w=8$ and length $l=800$, with the initial winding numbers $n=1$ (black) and $n=20$ (red). The solid lines represent $\Delta F^{\mathrm{PSS}}$ associated with the PSS solution calculated according to the (extended) LAMH theory, while the symbols represent $\Delta F^{\text {PSVAP }}$ associated with the PSVAP solution numerically obtained in the GL description (squares) and the London description (circles) by employing the string method.

clearly in Fig. 5(b), where $\varphi(x, y)$ is plotted as a function of $x$ for three $y$ levels (above the vortex, below the antivortex, and in between). These phase variations show that the phase change accumulated along the $+x$ direction will decrease/slip by $2 \pi$ once the VAP traverses the strip along the $y$ direction.

\section{Crossover from 1D PSS regime to 2D PSVAP regime}

In the presence of external magnetic field, the motion of the vortex relative to the antivortex is no longer along the $y$ direction. This field-induced distortion in the vortex passage indicates that the strip may accommodate the VAP with a transverse dimension smaller than the critical width at zero field $w_{c 0} \cong 8.8$. To verify this conjecture, we purposely study the saddle points for a strip of width $w=8$, which is slightly smaller than $w_{c 0}$. Figure 6 shows the free-energy barrier as a function of $\boldsymbol{\Phi}$ evaluated for transport current densities $j_{x}$ $\sim 0.02 j_{x c}$ and $j_{x} \sim 0.4 j_{x c}$, which correspond to the initial winding numbers $n=1$ and $n=20$ for a strip of length $l$ $=800$. The solid lines represent $\Delta F^{\mathrm{PSS}}$ associated with the PSS solution calculated according to the (extended) LAMH theory and numerically verified by using the string method. There are two sets of data presented for $\Delta F^{\text {PSVAP }}$ associated with the PSVAP solution. The squares represent the numeri-

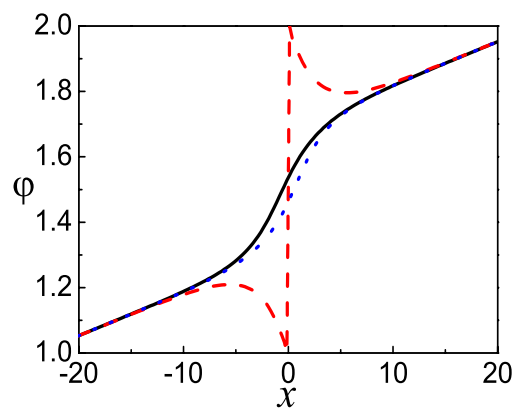

FIG. 5. (Color online) (a) Contour plot of the phase distribution $\varphi(x, y)$ (in the unit of $2 \pi$ ) in the PSVAP solution for $\boldsymbol{\Phi}=0.2$ [corresponding to state II in Fig. 3(c)]. The reference point of $\varphi=0$ is taken at $x=-l / 2$ and the color scale varies from blue for $\varphi=1$ to red for $\varphi=2$. (b) The phase variation in the $x$ direction, plotted for the three $y$ levels, marked by the horizontal lines in (a): $y=-6$ (black solid line), $y$ $=0$ (red dashed line), and $y=+6$ (blue dotted line). Only the segment of $-20 \leq x \leq 20$ is shown for clear illustration. 


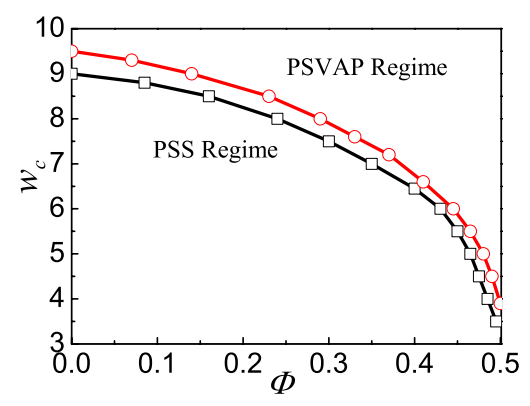

FIG. 7. (Color online) The critical width $w_{c}$ plotted as a function of $\boldsymbol{\Phi}$, evaluated for weak (black squares) and strong (red circles) transport current densities, realized by using the initial winding numbers $n=1$ and $n=20$ for the system of length $l=800$.

cal results obtained in the framework of GL theory and the circles represent those obtained in the framework of London theory. The core energy $E_{c}=1.25$ is used to make the London results match the GL results semiquantitatively, but with an observable deviation between the two data sets. This is expected for narrow strips in which the distance between the vortex and antivortex is too short to allow a satisfactory description by the London theory. The critical value of magnetic flux $\boldsymbol{\Phi}_{c}$, at which $\Delta F^{\mathrm{PSS}}=\Delta F^{\mathrm{PSVAP}}$, is found to be $\cong 0.26$ for $n=1$ and $\cong 0.30$ for $n=20$. Below $\boldsymbol{\Phi}_{c}$, although order-parameter variations along the $y$ direction are still allowed in computation, only those phase slips that occur through the 1D PSS solution are numerically found to exist. (That is, by evolving a string toward the stationary MEP, any order-parameter variation along the $y$ direction would be gradually removed and a PSS MEP is always reached in the end.) Above $\boldsymbol{\Phi}_{c}$, however, phase slips may occur through either the 1D PSS or the 2D PSVAP solution. The dominant contribution comes from those through the saddle point of lower free energy. Figure 6 shows that at the critical field $\boldsymbol{\Phi}_{c}$ we have $\Delta F^{\mathrm{PSVAP}}=\Delta F^{\mathrm{PSS}}$ within numerical error, and above $\boldsymbol{\Phi}_{c}$ we have $\Delta F^{\mathrm{PSS}}>\Delta F^{\mathrm{PSVAP}}$, i.e., phase slips occur dominantly through the 2D PSVAP solution. Comparing the critical fields for the two transport current densities, we find that a larger transport current density determines a stronger critical field. This is because for $w<w_{c 0} \cong 8.8$, the distorted vortex passage, which is necessitated by the existence of VAP, results from a competition between the field-induced persistent current in the transverse direction and the transport current in the longitudinal direction.

As noted in the beginning, the strip width used here, $w$ $=8$, is slightly smaller than the critical width $w_{c 0} \cong 8.8$ at zero field. Therefore, for $w=8$ the PSVAP solution is prohibited in the absence of external field but may be brought into existence by a sufficiently large external field. In other words, the critical width separating the 1D PSS regime and the 2D PSVAP regime is dependent on external field. Figure 7 shows the field dependence of the critical width $w_{c}$ for the weak and strong transport current densities used in Fig. 6. As expected, the critical width always decreases with increasing Ф. In particular, Fig. 7 shows that at the strongest external field $(\boldsymbol{\Phi}=0.5)$, phase slips can occur through the PSVAP solution in strips as narrow as $w \cong 3.5$ for the weak current or $w \cong 3.9$ for the strong current.

\section{CONCLUSION}

In the framework of GL theory, we have numerically calculated the saddle points and the associated free-energy barriers for the thermally activated phase slips in ultrathin DCSCs with transport current and external magnetic field both applied along the cylindrical axis. The hollow cylinder of radius $R$ is transformed into a $2 \mathrm{D}$ superconducting strip of width $w=2 \pi R$ with the PBC applied along the circumferential $(y)$ direction. The numerical results show the existence of a critical radius $R_{c}$ (or equivalently a critical width $w_{c}$ for the 2D strip). Below $R_{c}$, the DCSC behaves as a 1D wire with the phase slips described by the (extended) LAMH theory. Above $R_{c}$, the system recovers the $2 \mathrm{D}$ character with each phase slip realized through a thermally activated process of vortex-antivortex pair creation and annihilation. The effects of external magnetic field are quantitatively evaluated. The critical radius $R_{c}$ is found to decrease with increasing field strength, and hence applying a magnetic field may induce a 1D-to-2D transition in the phase-slip characteristics. It should be noted that the rate of phase slips (hence the resistance) is determined by both the prefactor $\Omega$ and the freeenergy barrier $\Delta F$. Here we have been focusing on the latter only as it always plays the dominant role through the exponential factor $e^{-\Delta F / k_{B} T}$.

We want to point out that our numerical results are presented for the dimensionless system (defined in Sec. II). For a real DCSC the temperature dependence of free-energy barrier comes directly from the temperature-dependent units through which relevant dimensionless parameters are defined. Obviously, our numerical study does not give a complete prediction for the temperature dependence of resistance. However, in the limit of weak transport current, the influence arising from the temperature-dependent unit of current density can be neglected, and hence the dominant temperature effect comes from the unit $G(T) \propto T_{c}-T$ for free energy and the dimensionless radius of DCSC $R(T) \propto\left(T_{c}\right.$ $-T)^{1 / 2}$, which lead to the decrease in free-energy barrier and hence the increase in resistance $\left(\propto \exp \left[-G(T) \Delta F / k_{B} T\right]\right)$ as $T_{c}$ is approached from below. For relatively large DCSCs (with $R>R_{c}$ at $T$ well below $T_{c}$ ), the temperature dependence in $R(T) \propto\left(T_{c}-T\right)^{1 / 2}$ indicates a crossover temperature $T^{*}$ that separates the 1D PSS and 2D PSVAP regimes. Above $T^{*}$ (but still below $T_{c}$ ), the resistance follows the description by the (modified) LAMH theory. Below $T^{*}$, however, the system enters into the $2 \mathrm{D}$ regime and the resistance becomes larger than that predicted by the LAMH theory because of the reduced PSVAP barrier. For the difference between the two regimes, as far as temperature dependence is concerned, we emphasize that the difference in dependence on the dimensionless width $w(T)=2 \pi R(T)$ (linear for PSS solution vs logarithmic for PSVAP solution) plays the dominant role. Furthermore, as the external field is gradually increased, $T^{*}$ can be shifted toward higher temperatures because smaller dimensionless critical width $\left(w_{c}\right.$ in Fig. 7$)$ corresponds to larger length unit and thus temperature closer to $T_{c}$. Given the large radii $\left(2 \pi R>w_{c}\right)$ required for PSVAP activation to occur, one may wonder whether the free-energy barrier is already too large to produce a measurable resistance (i.e., the rate $\sim e^{-\Delta F / k_{B} T}$ is too small to contribute to a measurable 
signal). To see whether the 1D-to-2D crossover is observable (presumably for relatively large radii and low temperatures), here we make a comparison as follows. Consider a $1 \mathrm{D}$ superconducting whisker of radius $R_{\text {whi }}$ and a DCSC of radius $R_{\text {cyl }}$ and thickness $d$. The free-energy barrier for the whisker is given by $a\left(\pi R_{\text {whi }}^{2}\right) \xi \alpha^{2} / \beta$ while that for the DCSC is given by $b\left(2 \pi R_{\text {cyl }} d\right) \xi \alpha^{2} / \beta$. Here $\pi R_{\text {whi }}^{2}$ and $2 \pi R_{\text {cyl }} d$ are the crosssectional areas of the whisker and the DCSC, respectively, $\alpha^{2} / \beta$ is the energy density unit, $\xi$ is the correlation length, and $a$ and $b$ are two numerical values. Let the whisker be a $1 \mathrm{D}$ system with $R_{\text {whi }}=\xi / 2$ and the DCSC be close to the crossover dimension with $2 \pi R_{\mathrm{cyl}} \approx 8.8 \xi$ and thus $b \approx a$. The ratio of the free-energy barrier for DCSC to that for whisker becomes $2 \pi R_{\text {cyl }} d / \pi R_{\text {whi }}^{2} \approx 11 d / \xi$. Therefore, if the thickness $d$ is made small enough $(d \sim 0.1 \xi)$, then the free-energy barrier for DCSC close to the crossover is of the same order as that for a 1D whisker whose resistance due to phase slips is considered measurable. Finally, we note that our results show no signal for the peculiar temperature steps of resistance described in Refs. 21 and 22, whose origin calls for further theoretical and experimental works.

\section{ACKNOWLEDGMENT}

This work was partially supported by the Hong Kong RGC under Grant No. HKUST602904.

\section{APPENDIX}

Without lengthy yet straightforward derivations, here we present the analytical expressions for both the PSS solution in the extended LAMH theory and the PSVAP solution based on the London theory ${ }^{28-30}$ for DCSCs in the presence of external magnetic field and transport current, both applied along the cylindrical axis. Mathematically, a hollow cylinder of radius $R$ is transformed into a $2 \mathrm{D}$ strip of width $w=2 \pi R$ with PBC applied in the transverse $y$ direction, as illustrated in Fig. 1. For consistency the dimensionless quantities defined in Sec. II are used.

\section{PSS solution in the extended LAMH theory}

In the extended LAMH theory, the PSS solution $\psi_{S}^{\text {PSS }}$ is expressed as

$$
\begin{aligned}
\psi_{s}^{\mathrm{PSS}}(x)= & \left\{\sqrt{1-A^{2}-3 k_{s}^{2}} \tanh \left[\sqrt{\left(1-A^{2}-3 k_{s}^{2}\right) / 2} x\right]\right. \\
& \left.-i \sqrt{2} k_{s}\right\} e^{i k_{s} x}
\end{aligned}
$$

where $k_{s}$ is a wave vector determined by

$$
k_{s}=k_{n}-(2 / l) \tan ^{-1} \sqrt{\left(1-A^{2}-3 k_{s}^{2}\right) / 2 k_{s}^{2}},
$$

which satisfies $k_{n-1}<k_{s}<k_{n}$. From the explicit expressions for $\psi_{n}$ and $\psi_{s}^{\mathrm{PSS}}$, the free-energy barrier $\Delta F^{\mathrm{PSS}}=F\left[\psi_{s}^{\mathrm{PSS}}\right]$ $-F\left[\psi_{n}\right]$ can be readily obtained as follows:

$$
\begin{aligned}
\Delta F^{\mathrm{PSS}}= & w\left[\frac{2\left(1-A^{2}\right) \sqrt{2\left(1-A^{2}-3 k_{s}^{2}\right)}}{3}\right. \\
& \left.+\frac{l\left(k_{s}^{2}-k_{n}^{2}\right)\left(2-2 A^{2}-k_{n}^{2}-k_{s}^{2}\right)}{4}\right],
\end{aligned}
$$

which is proportional to the circumference of DCSC as expected from the 1D character of the PSS solution. The original LAMH results can be recovered from Eqs. (A1)-(A3) as the vector potential $A$ goes to zero. ${ }^{26,27}$ Although not the focus of this paper, it is worth mentioning some limiting behaviors, which might be instructive to experiments. In the limit of weak transport current, $k_{n} \rightarrow 0$, then $k_{s} \cong(2 n-1) \pi / l$ to the leading order, and hence $\Delta F^{\mathrm{PSS}} \cong w\left[2\left(1-A^{2}\right)\right]^{3 / 2} / 3$, which gives $\Delta F^{\mathrm{PSS}} \cong w \sqrt{2}\left(2 / 3-A^{2}\right)$ in the limit of weak field $(A \rightarrow 0)$.

\section{PSVAP solution in the London theory}

Considering the translational invariance of the VAP energy due to the PBCs applied in the $x$ and $y$ directions, we simply assume the VAP to be generated in the origin. In the presence of a current of density $\mathbf{j}=f^{2} \mathbf{k} \cong \mathbf{k}$, where $f \cong 1$ for weak currents, $k_{x}=k_{n}$ for the transport current, and $k_{y}=-A$ for the field-induced persistent current, the energy of a VAP in a strip of width $w$ can be expressed as

$$
\begin{aligned}
F\left(\mathbf{r}_{R}\right)= & \pi \ln \left\{(w / \pi)^{2}\left[\sin ^{2}\left(\pi y_{R} / w\right)+\sinh ^{2}\left(\pi x_{R} / w\right)\right]\right\} \\
& -2 \pi\left(k_{x} y_{R}-k_{y} x_{R}\right)+2 E_{c},
\end{aligned}
$$

which is a function of the position of the vortex relative to the antivortex, $\mathbf{r}_{R}=\left(x_{R}, y_{R}\right)$. In the right-hand side of Eq. (A4), the first and second terms come from the vortexantivortex interaction and vortices-current interaction, respectively, and $E_{c}$ in the third term is the positive vortex core energy, usually treated as an adjustable parameter. ${ }^{16}$ Note that as $\mathbf{r}_{R} \rightarrow(0,0)$ or $(0, w), F \rightarrow-\propto$ in approaching the $\psi_{n}$ state or the $\psi_{n-1}$ state. Equation (A4) becomes invalid as the vortex-antivortex distance approaches $\sim 1$ because the size of the vortex core has not been taken into account. The saddle point $\mathbf{r}_{s}=\left(x_{s}, y_{s}\right)$, i.e., the PSVAP solution in real space, satisfies the stationary condition $\nabla F\left(\mathbf{r}_{s}\right)=0$, i.e.,

$$
\left.\frac{w}{\pi^{2}} \frac{\partial F}{\partial x_{R}}\right|_{\mathbf{r}_{s}}=\frac{\sinh \left(2 \pi x_{s} / w\right)}{\sin ^{2}\left(\pi y_{s} / w\right)+\sinh ^{2}\left(\pi x_{s} / w\right)}+\frac{2 w k_{y}}{\pi}=0
$$

and

$$
\left.\frac{w}{\pi^{2}} \frac{\partial F}{\partial y_{R}}\right|_{\mathbf{r}_{s}}=\frac{\sin \left(2 \pi y_{s} / w\right)}{\sin ^{2}\left(\pi y_{s} / w\right)+\sinh ^{2}\left(\pi x_{s} / w\right)}-\frac{2 w k_{x}}{\pi}=0,
$$

which can be solved numerically. To find the passage for the motion of the vortex relative to the antivortex in a thermally activated phase slip, the string method has been employed to obtain the MEP in real space for $F\left(\mathbf{r}_{R}\right)$. The free-energy barrier $\Delta F^{\mathrm{PSVAP}}=F\left(\mathbf{r}_{s}\right)$ can be obtained by locating the maximum of $F\left(\mathbf{r}_{R}\right)$ along the MEP. Analytical expressions for the PSVAP solution can be obtained in the following special cases:

(1) In the absence of external field, $k_{y}=0$, and the saddle point is given by $x_{s}=0$ (vortex passage along the transverse direction) and $y_{s}=(w / \pi) \tan ^{-1}\left(\pi / w k_{x}\right)$, with the corresponding energy barrier 
$\Delta F^{\mathrm{PSVAP}}=2 \pi\left[\ln \frac{w}{\pi \sqrt{1+\left(w k_{x} / \pi\right)^{2}}}-\frac{w k_{x}}{\pi} \tan ^{-1}\left(\frac{\pi}{w k_{x}}\right)\right]+2 E_{c}$.

Therefore, $\Delta F^{\mathrm{PSVAP}}\left(k_{x}, w\right)=2 \Delta F^{\mathrm{PSV}}\left(k_{x}, w / 2\right)$, where $\Delta F^{\mathrm{PSV}}$ is the free-energy barrier associated with the PSV solution for a strip of width $w / 2$ with superconductor-insulator boundary condition [see Eq. (A3) in Ref. 16]. This relation is a direct consequence of the mapping introduced in Sec. II B for zero field: a strip of width $w$ with $\mathrm{PBC}$ in the $y$ direction is equivalent to two strips of width $w / 2$ with superconductor- insulator boundary condition in the same direction. Furthermore, in the limit of $k_{x} \rightarrow 0, \Delta F^{\mathrm{PSVAP}} \cong-\pi w k_{x}+\left(w^{2} / \pi\right) k_{x}^{2}$ $+2 \pi \ln (w / \pi)+2 E_{c}$.

(2) In the limit of $k_{x} \rightarrow 0$ and $k_{y} \rightarrow 0$, the saddle point $\mathbf{r}_{s}$ can be approximated by $x_{s} \cong-(w / \pi)^{2} k_{y}$ and $y_{s} \cong w / 2$ $-(w / \pi)^{2} k_{x}$, which lead to the free-energy barrier $\Delta F^{\text {PSVAP }}$ $\cong-\pi w k_{x}+\left(w^{2} / \pi\right)\left(k_{x}^{2}-k_{y}^{2}\right)+2 \pi \ln (w / \pi)+2 E_{c}$. Therefore, the response of the free-energy barrier to weak field in the limit of zero transport current is a net change of $-k_{y}^{2} w^{2} / \pi$ $=-A^{2} w^{2} / \pi$.
*Author to whom correspondence should be addressed; maqian@ust.hk

${ }^{1}$ W. A. Little, Phys. Rev. 156, 396 (1967).

${ }^{2}$ J. S. Langer and V. Ambegaokar, Phys. Rev. 164, 498 (1967).

${ }^{3}$ D. McCumber and B. Halperin, Phys. Rev. B 1, 1054 (1970).

${ }^{4}$ J. M. Kosterlitz and D. J. Thouless, J. Phys. C 6, 1181 (1973).

${ }^{5}$ M. R. Beasley, J. E. Mooij, and T. P. Orlando, Phys. Rev. Lett. 42, 1165 (1979).

${ }^{6}$ S. Doniach and B. A. Huberman, Phys. Rev. Lett. 42, 1169 (1979).

${ }^{7}$ B. I. Halperin and D. R. Nelson, J. Low Temp. Phys. 36, 599 (1979).

${ }^{8}$ L. A. Turkevich, J. Phys. C 12, L385 (1979).

${ }^{9}$ P. Minnhagen, Phys. Rev. B 24, 6758 (1981).

${ }^{10}$ A. M. Kadin, K. Epstein, and A. M. Goldman, Phys. Rev. B 27, 6691 (1983).

${ }^{11}$ J. E. Lukens, R. J. Warburton, and W. W. Webb, Phys. Rev. Lett. 25, 1180 (1970).

${ }^{12}$ R. S. Newbower, M. R. Beasly, and M. Tinkham, Phys. Rev. B 5, 864 (1972).

${ }^{13}$ S. Michotte, S. Matefi-Tempfli, L. Piraux, D. Y. Vodolazov, and F. M. Peeters, Phys. Rev. B 69, 094512 (2004).

${ }^{14}$ A. Verevkin, J. Zhang, R. Sobolewski, A. Lipatov, O. Okunev, G. Chulkova, A. Korneev, K. Smirnov, G. N. Goltsman, and A. Semenov, Appl. Phys. Lett. 80, 4687 (2002).
${ }^{15}$ M. Bell, A. Sergeev, V. Mitin, J. Bird, A. Verevkin, and G. Gol'tsman, Phys. Rev. B 76, 094521 (2007).

${ }^{16}$ C. Qiu and T. Qian, Phys. Rev. B 77, 174517 (2008).

${ }^{17}$ W. A. Little and R. D. Parks, Phys. Rev. Lett. 9, 9 (1962).

${ }^{18}$ R. D. Parks and W. A. Little, Phys. Rev. 133, A97 (1964).

${ }^{19}$ R. P. Groff and R. D. Parks, Phys. Rev. 176, 567 (1968).

${ }^{20}$ Y. Liu, Yu. Zadorozhny, M. M. Rosario, B. Y. Rock, P. T. Carrigan, and H. Wang, Science 294, 2332 (2001).

${ }^{21}$ H. Wang, M. M. Rosario, N. A. Kurz, B. Y. Rock, M. Tian, P. T. Carrigan, and Y. Liu, Phys. Rev. Lett. 95, 197003 (2005).

${ }^{22}$ Y. Liu, H. Wang, Yu. Zadorozhny, M. M. Rosario, B. Y. Rock, N. A. Kurz, and P. T. Carrigan, Physica C 468, 331 (2008).

${ }^{23}$ W. E, W. Ren, and E. Vanden-Eijnden, Phys. Rev. B 66, 052301 (2002).

${ }^{24}$ W. E, W. Ren, and E. Vanden-Eijnden, J. Appl. Phys. 93, 2275 (2003).

${ }^{25}$ W. E, W. Ren, and E. Vanden-Eijnden, J. Chem. Phys. 126, 164103 (2007).

${ }^{26}$ T. Qian, W. Ren, and P. Sheng, Phys. Rev. B 72, 014512 (2005).

${ }^{27}$ C. Qiu, T. Qian, and W. Ren, Phys. Rev. B 77, 104516 (2008).

${ }^{28}$ J. Pearl, Appl. Phys. Lett. 5, 65 (1964).

${ }^{29}$ V. G. Kogan, Phys. Rev. B 49, 15874 (1994).

${ }^{30}$ F. Tafuri, J. R. Kirtley, D. Born, D. Stornaiuolo, P. G. Medaglia, P. Orgiani, G. Balestrino, and V. G. Kogan, Europhys. Lett. 73, 948 (2006). 